\title{
Planning and designing a self-compassion intervention for family carers of people living with dementia: a person-based and co-design approach
}

\author{
Jenny Murfield ${ }^{1,2,3^{*}} \mathbb{B}$, Wendy Moyle $e^{2,3}$ and Analise $\mathrm{O}^{\prime}$ Donovan ${ }^{4}(\mathbb{C})$
}

\begin{abstract}
Background: This article describes the research activities undertaken to plan and design a self-compassion intervention for family carers of people living with dementia using a person-based and co-design approach. In providing this example, our aim is two-fold: to highlight the value of using qualitative research and co-design processes within intervention development; and to showcase systematic reporting of an intervention's early planning and design stages.

Methods: A person-based and co-design approach informed the planning and design of the self-compassion intervention. In Stage 1, qualitative interviews were undertaken with 14 family carers of people living with dementia and 14 professional stakeholders. In Stage 2, intervention guiding principles were developed, psychological theory was incorporated, and six family carers of people living with dementia were engaged as co-designers.

Results: Knowledge generated during intervention planning identified that the intervention should be situated within the concept of compassion more broadly; address misperceptions, fears, blocks, and resistances to self-compassion; and target feelings of shame, guilt, and self-criticism. Subsequent intervention design activities determined that the needs of family carers of people living with dementia were best met by tailoring an existing intervention, namely group-based Compassion-Focused Therapy.

Conclusions: Our systematic approach highlights the value of incorporating in-depth qualitative research and codesign within the intervention development process to prioritise the perspectives and lived experiences of family carers of people living with dementia. The planning and design process outlined provides insight that is applicable to the development of our intervention and complex health interventions within gerontology and beyond.

Keywords: Alzheimer's disease, Caregivers, Co-design, Dementia, Intervention development, Mental health, Patient and public involvement, Person-based approach, Qualitative research, Self-compassion
\end{abstract}

*Correspondence: jenny.murfield@deakin.edu.au

${ }^{1}$ Food \& Mood Centre, IMPACT (Institute for Mental and Physical Health and Clinical Translation), School of Medicine, Deakin University, Geelong, Australia

Full list of author information is available at the end of the article

\section{Introduction}

Family Carers of People Living with Dementia

Providing informal care to a family member living with dementia can be a positive experience, and many carers report feelings of satisfaction and personal reward [1]. However, it can also be challenging, and it is well-established that family carers can be impacted in numerous ways [2]. In terms of psychological and emotional health, 
studies show that family carers of people living with dementia can experience greater psychological distress than caregivers of other conditions $[3,4]$. As many as one in three family carers of people living with dementia experience depression, and one in two report subjective burden [5]. Feelings of guilt and shame are also common within the dementia caregiving role, and heightened levels of both have been associated with the development of depressive symptoms [6,7].

To help ameliorate some of the described negative impacts, the last few decades have seen an increased focus on the development and testing of different psychosocial interventions to support family carers of people living with dementia [8]. Meta-analyses and reviews have documented encouraging effects for some interventions within clinical trials, and particularly for those that assume a cognitive behavioural approach [8-10]. Most recently, this has included a focus on modern approaches that target mindfulness, acceptance, and compassion, both for the self and others $[11,12]$.

\section{Self-Compassion}

Self-compassion is variously defined within the literature. In Neff's [13] conceptualisation, it is understood as treating yourself with care during times of suffering and involves self-kindness rather than self-judgement; common humanity rather than isolation; and mindfulness rather than over-identification. In Gilbert's [14] understanding, self-compassion is defined within the concept of compassion more broadly, being regarded as part of a three-way 'flow' (involving compassion for self, to others, and from others), which involves two aspects: a sensitivity to suffering and a commitment to prevent and/ or alleviate that suffering. Drawing on these definitions, research conducted within various clinical and non-clinical populations has found positive links between selfcompassion and psychological wellbeing [15] and has shown that self-compassion can be cultivated to improve psychological health [16]. Within dementia family caregiving specifically, similar promising findings have been demonstrated in cross-sectional studies [17, 18], and in a published group intervention study of CompassionFocused Therapy for couples with a dementia diagnosis [19]. Nevertheless, this understanding is limited to a handful of studies and, as yet there is no self-compassion intervention available for specific use with family carers of people living with dementia.

\section{Intervention Development}

Despite scientific promise, limited numbers of dementia caregiver interventions are translated into real-world use $[10,20]$, and inadequate reporting of the intervention development process $[10,20]$ and insufficient understanding of the carers' needs [e.g., 21] have been implicated. Consistent with health intervention research at large $[22,23]$, these findings suggest that the development of new and/or alternative interventions to support family carers of people living with dementia should be systematically described and grounded in an in-depth, qualitative understanding of users' real-life needs and preferences. The Person-Based Approach (PBA) to intervention planning and development [24-26], and Patient and Public Involvement (PPI) through a process of codesign [27-29], offer ideal frameworks to achieve this.

The PBA utilises extensive in-depth qualitative research to situate the intervention in the perspective and lived experience of the people who will use it [2426]. Although a relatively new approach, it has been successfully used to develop various health interventions for different populations, including family carers [30] and older adults [31,32]. Offering a flexible approach to intervention development, the PBA involves a qualitative exploration of the key issues, needs, and challenges that the intervention must address, and the formulation of guiding principles that set out the intervention's key design objectives and their corresponding key features. The approach can be used alongside traditional evidenceand theory-based intervention development frameworks, including the UK Medical Research Council's (MRC) guidance [33, 34]. It is also advocated for use with methods of PPI, including co-design [35].

Defined as doing research 'with or by' public and patients rather than doing it 'to, about, or for' them [28, 29], PPI is universally acknowledged as a valuable and important part of the research process that can result in the production of interventions that have greater relevance to everyday practice [36]. Within dementia research, PPI is rapidly increasing [37], and a growing number of intervention development studies are involving family carers and people living with dementia in the planning and design stages as co-designers [37-39]. Nonetheless, the processes by which researchers have undertaken co-design are not well documented, and greater transparency in reporting is needed to facilitate best practices [37].

\section{The Current Study}

Building on the described work, we sought to develop a self-compassion intervention for family carers of people living with dementia. The purpose of this article is to describe the research activities we undertook to plan and design this intervention using a personbased [24-26] and co-design approach [27-29]. By providing this example, we aim to highlight the value of using qualitative research and co-design to prioritise the perspectives and lived experiences of the 
intervention's intended target users, and to showcase a systematic approach to the early planning and designing stages within intervention development. To promote rigour in our reporting, we have used the Guidelines for the Reporting of Intervention Development Checklist (GUIDED) [40] and the Guidance for Reporting Involvement of Patients and the Public (GRIPP2-SF) [41] (see Additional file 1).

\section{Methods}

\section{Intervention Planning and Design Process}

The complete intervention development process used evidence-based, theory-based, person-based, and codesign approaches to inform the planning and design of the self-compassion intervention [24-29, 33, 34]. Although not described within this article, we first drew on the 'development' phase of the MRC framework for best practice in developing and evaluating complex health interventions [33, 34]. In brief, this involved three evidence- and theory-based activities: (1) literature reviews [12, 42]; (2) conceptual analysis [43]; and (3) cross-sectional survey study [18, 44]. Relevant to this article's focus, we then chose to supplement the MRC framework with additional qualitative and co-design approaches to ensure that the intervention was grounded in the perspective and lived experience of the intervention's target users. For this, we first drew on the 'planning' and 'design' phases of the PBA for developing behavioural interventions [24-26]. We then incorporated the principles of PPI in health and medical research [27] by using a co-design process that broadly aligned with the deciding how to do it'/'designing and managing' phase of the research cycle $[28,29]$.

The person-based and co-design approach reported within this article comprised two research stages that focused on (1) intervention planning, and (2) intervention design. In Stage 1, we undertook qualitative interviews with family carers of people living with dementia and professional stakeholders to explore perceptions, barriers, facilitators, and contextual issues pertinent to planning the intervention. In Stage 2, we undertook a co-design process that saw us engage a small group of family carers of people living with dementia to assist in decision-making about the intervention's design, including the development of guiding principles, the incorporation of psychological theory, and the creation of fictional scenarios and personas.

We received ethical approval for the study from Griffith University (Ref: 2019/481), and we obtained written informed consent and verbal assent from qualitative participants and co-design group members.

\section{Stage 1 Intervention Planning: Qualitative Interviews}

To explore perspectives about the proposed intervention and identify any potential barriers, facilitators, and contextual issues relevant to its design, we conducted semi-structured interviews with 14 family carers of people living with dementia (aged $\geq 18$ years and self-identifying as a family carer of a person living with dementia) and 14 professional stakeholders (academic clinicians with expertise in ageing/dementia, family caregiving, and/or compassion, and carer support professionals). We recruited family carers of people living with dementia from Australia using convenience sampling. This involved participants voluntarily responding to social media posts, promotions in carer organisations' electronic newsletters, and in-person talks at carer support groups (within $60 \mathrm{kms}$ of Brisbane, Queensland). We recruited professional stakeholders using purposive sampling. This involved the lead author emailing professional contacts with known relevant expertise from Australia and the UK.

Between September and December 2019, the lead author conducted individual, one-off, verbal, semistructured interviews with participants. Interviews were conducted either by telephone $(n=16)$, via videoconferencing $(n=9)$, or in-person $(n=3)$ and averaged $30 \mathrm{~min}$ utes in duration (range $15-62 \mathrm{~min}$ ). We used three interview schedules that we tailored slightly to accommodate participant groups' differing contexts. However, across all interviews, we asked participants a core set of questions about their understanding of self-compassion as a concept; their thoughts about a self-compassion intervention for family carers of people living with dementia; and the things that they thought might help or hinder intervention implementation, including methods and modes of delivery. We digitally audio-recorded interviews and transcribed them verbatim, and we made analytical field notes. To analyse the data, we used a recursive process of inductive, reflexive thematic analysis $[45,46]$. This involved: (1) repeatedly listening to and reading the transcripts to become familiar with the data; (2) line-byline coding on hard-copies of transcripts to generate initial codes; (3) grouping codes with a shared meaning to generate initial themes and subthemes; (4) reviewing the developing themes and subthemes for meaning against the study's aim; and (5) defining the developed themes using supporting quotations from the data. The lead author undertook this analysis and met with the authorship team multiple times to discuss the developing coding frame and to reflect on their interpretative judgement of the themes identified in relation to the aims of the analysis [47]. Consistent with the assumptions of reflexive thematic analysis [48], the total number of interviews we conducted was pragmatically determined, seeing 
the interviews individually and collectively reviewed for their adequacy (i.e., richness and complexity) to meet the study's aims.

\section{Stage 2 Intervention Design: Co-Design Group}

To ensure that the design of the proposed self-compassion intervention was best suited to the real-life needs and preferences of its intended target users, we engaged six family carers of people living with dementia to work alongside the first author as co-designers. Using convenience sampling, we recruited co-design members $(\geq 18$ years and self-identifying as a family carer of a person living with dementia) from Australia. This involved the lead author posting advertisements on social media and emailing/writing to known family carers of people living with dementia. We did not require co-design members to have any experience or training in research methods; however, all had participated in at least one research project previously, independent of this study and the authors.

Between October and November 2020, all co-design members took part in four, 90-minute sessions that were conducted weekly. Five of the co-design members took part as a group and participated in the sessions online using Microsoft Teams videoconferencing. One codesign member opted to participate individually, as based on their preference to engage independently rather than in a group. For each week's session, this involved them watching a pre-recorded video that mirrored the same content as the online session, and then participating in a follow-up telephone conversation. In keeping with recommendations for PPI within dementia care research [39], we financially reimbursed all co-design members for their involvement (AUD132 per session).

The lead author was trained in conducting qualitative and group-based discussions with this population and facilitated all sessions. Although we used a session agenda, we were flexible and adopted an iterative approach by covering content in sessions as necessary. Sessions were video and/or digitally audio-recorded and collectively covered the following content: discussion, feedback, and refinement of the intervention's formulated guiding principles; discussion and feedback on the proposed structure and broad content of the proposed intervention; and the creation of fictional caregiver scenarios and personas for use in the intervention.

\section{Results}

\section{Stage 1 Intervention Planning: Qualitative Interviews}

From the 28 interviews we conducted exploring the potential barriers, facilitators, and contextual issues pertinent to the intervention's design, we inductively identified five relevant themes and 12 sub-themes. Given that the purpose of this article is to describe the intervention development process, we have chosen to present participant characteristics (Table 1) and a selection of de-identified participant quotations to support the final themes (Table 2) within a tabular form, rather than including these data within the text directly.

\section{Understanding of Self-Compassion}

Self-compassion was a largely unknown concept to participants. Some family carers of people living with dementia had never heard of self-compassion before, and a minority of professional stakeholders with expertise in ageing and dementia were unfamiliar with the concept. When describing what they understood self-compassion to be, it was common for participants to situate self-compassion within the concept of compassion as it related to others more broadly (i.e., giving and receiving). Specifically, some of the family carers of people living with dementia we interviewed had never considered giving compassion to themselves and only understood compassion as relating to caring for another person. Other comments from participants reflected the bidirectional relationships between self-compassion and compassion for others (i.e., the importance of caring for the self in order to care well for another/caring for another as a way of caring for the self), as well as the relationship between self-compassion and being open to receiving compassion from others (i.e., accepting and bringing in outside help).

\section{Perceptions of Self-Compassion}

In the main, participants perceived self-compassion positively, describing potential benefits for carers in helping with healthy emotion regulation; reducing self-criticism and feelings of guilt; enhancing resilience; enabling selfadvocacy; and enhancing carers' ability to self-evaluate. That said, some participants also added important qualifiers: self-compassion should not be used to either excuse or allow psychological or physical abuse within a caregiving relationship, or for a family carer to remain in an acutely stressful situation.

One family carer of a person living with dementia was overtly critical of self-compassion, seeing it as self-indulgent and focused solely and unnecessarily on the self. Although personally supportive of the concept, other family carers of people living with dementia also commented that self-compassion could be interpreted as selfish, self-indulgent, and related to the self-care rhetoric commonly heard in dementia carer support services and the wider general discourse. These sentiments were echoed by professional stakeholders, whereby it was considered likely that some family carers of people living with dementia may be resistant to self-compassion due to associations with self-indulgence, self-pity, and weakness. 
Table 1 Characteristics of qualitative interview participants and co-design group members

Characteristic

Descriptive statistics

\section{STAGE 1: QUALITATIVE INTERVIEWS}

Family carers of people living with dementia $(n=14)$

Age (years) ${ }^{a}$

$62.5(14.4)$

Identifying gender (Female: Male) ${ }^{\text {b }}$

Country of residence (Australia) ${ }^{b}$

In employment (Yes: No) ${ }^{b}$

Relationship to care recipient (Partner: Offspring) $)^{b}$

Length of time caring for care recipient (years) ${ }^{a}$

11 (78.6): 3 (21.4)

$14(100)$

4 (28.6): $10(71.4)$

$7(50): 7(50)$

Age of care recipient (years)

6.5 (2.9)

$78.6(10.4)$

Identifying gender of care recipient (Female: Male) ${ }^{b}$

9 (64.3): 5 (35.7)

Care recipient's type of dementia ${ }^{b}$

Alzheimer's disease

$7(50)$

Unspecified

4 (28.6)

Frontotemporal

$1(7.1)$

Lewy-body

$1(7.1)$

Vascular

$1(7.1)$

Professional stakeholders ( $n=14$ )

Academic clinicians with expertise in ageing \& dementia $(n=5)$

Identifying gender (Female: Male) ${ }^{b}$

4 (80): $1(20)$

Country of residence (Australia) ${ }^{\mathrm{b}}$

$5(100)$

Roleb

Clinical psychologist

Mental health nurse

$1(20)$

Occupational therapist

$1(20)$

Old age psychiatrist

$1(20)$

Social gerontologist

1 (20)

Academic clinicians with expertise in compassion $(n=4)$

Identifying gender (Female: Male) ${ }^{\mathrm{b}}$

$2(50): 2(50)$

Country of residence (Australia: UK) ${ }^{b}$

Role $^{b}$

Clinical psychologist

$3(75)$

$1(25)$

Carer support professionals $(n=5)$

Identifying gender (Female: Male) ${ }^{b}$

$4(80): 1(20)$

Country of residence (Australia) ${ }^{b}$

$5(100)$

Role $^{b}$

Counsellor

$2(40)$

Senior management (education, training, \& improvement)

$2(40)$

Educator

1 (20)

\section{STAGE 2: CO-DESIGN GROUP}

Family carers of people living with dementia $(n=6)$

Age (years) ${ }^{a}$

$61(8.0)$

Identifying gender (Female: Male) ${ }^{\mathrm{b}}$

5 (83.3): 1 (16.7)

$6(100)$

$3(50): 3(50)$

1 (16.7): 5 (83.3)

Relationship to care recipient (Partner: Offspring) ${ }^{\mathrm{b}}$

$5.8(2.8)$

Length of time caring for care recipient (years)

$86(9.2)$

Age of care recipient (years)

$4(66.7): 2(33.3)$

Identifying gender of care recipient (Female: Male)

$4(66.7): 2(33.3)$

Note. ${ }^{\mathrm{a}}$ continuous variables are reported as $M(S D) ;{ }^{\mathrm{b}}$ categorical variables are reported as $n(\%)$ 


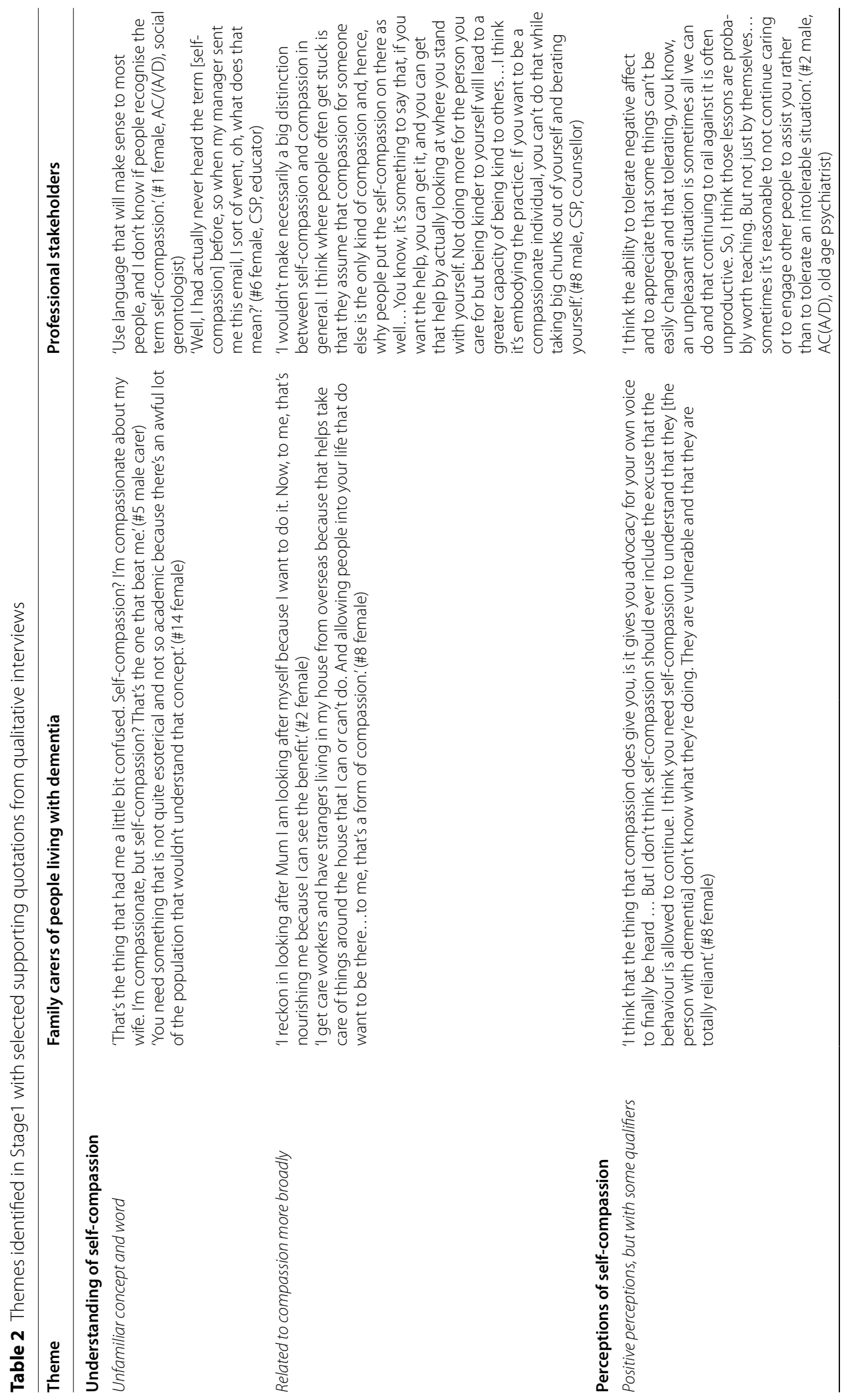




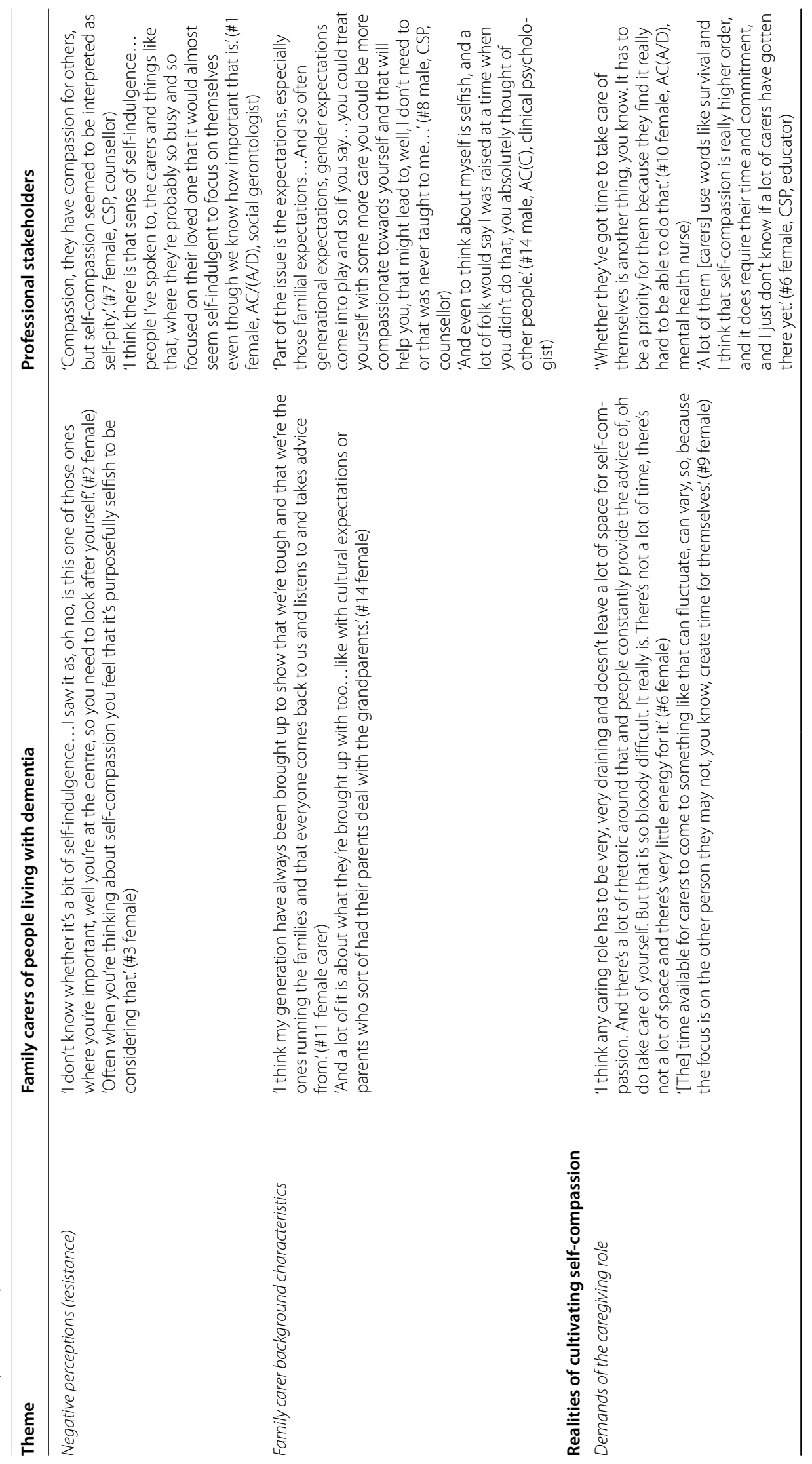




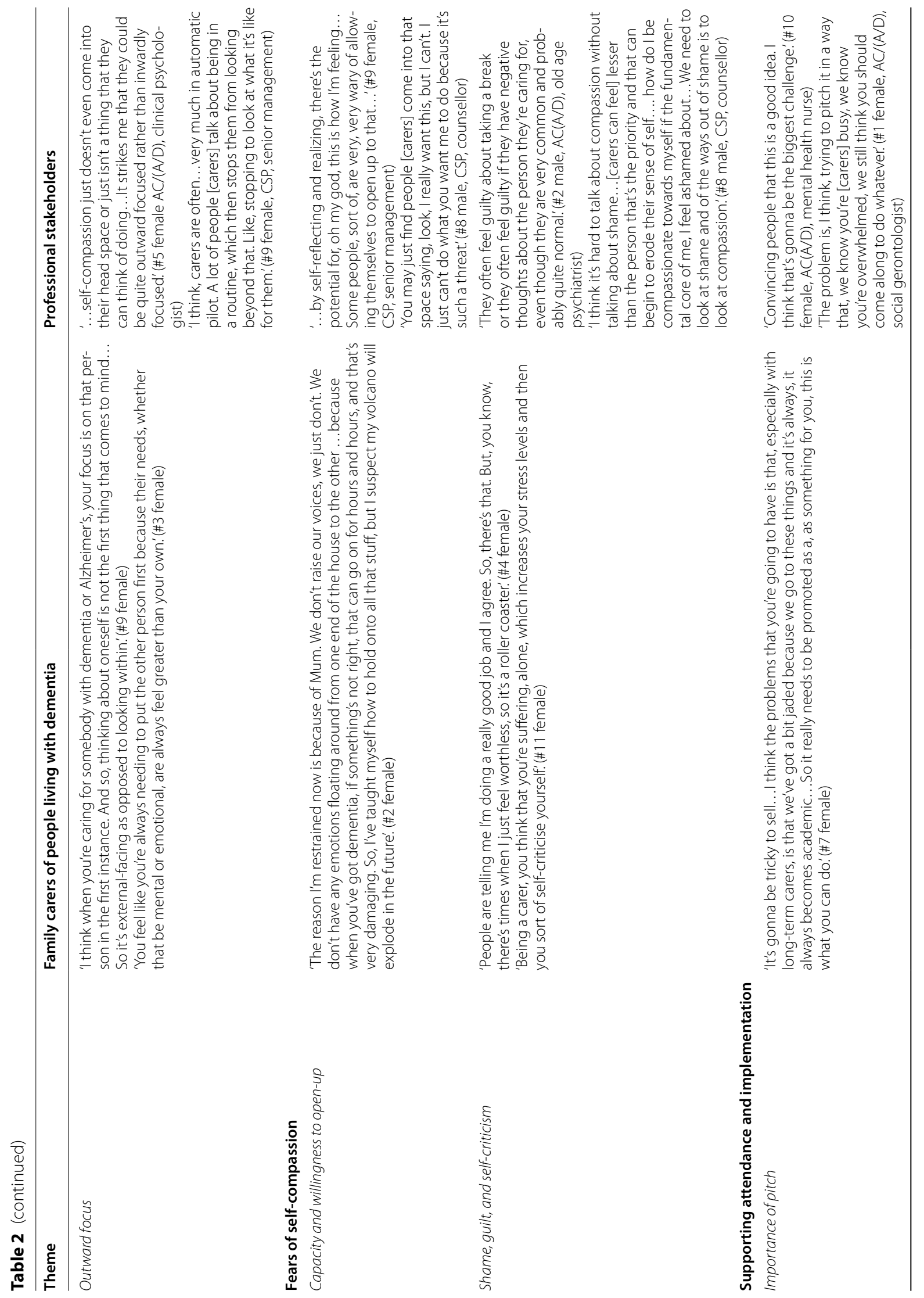




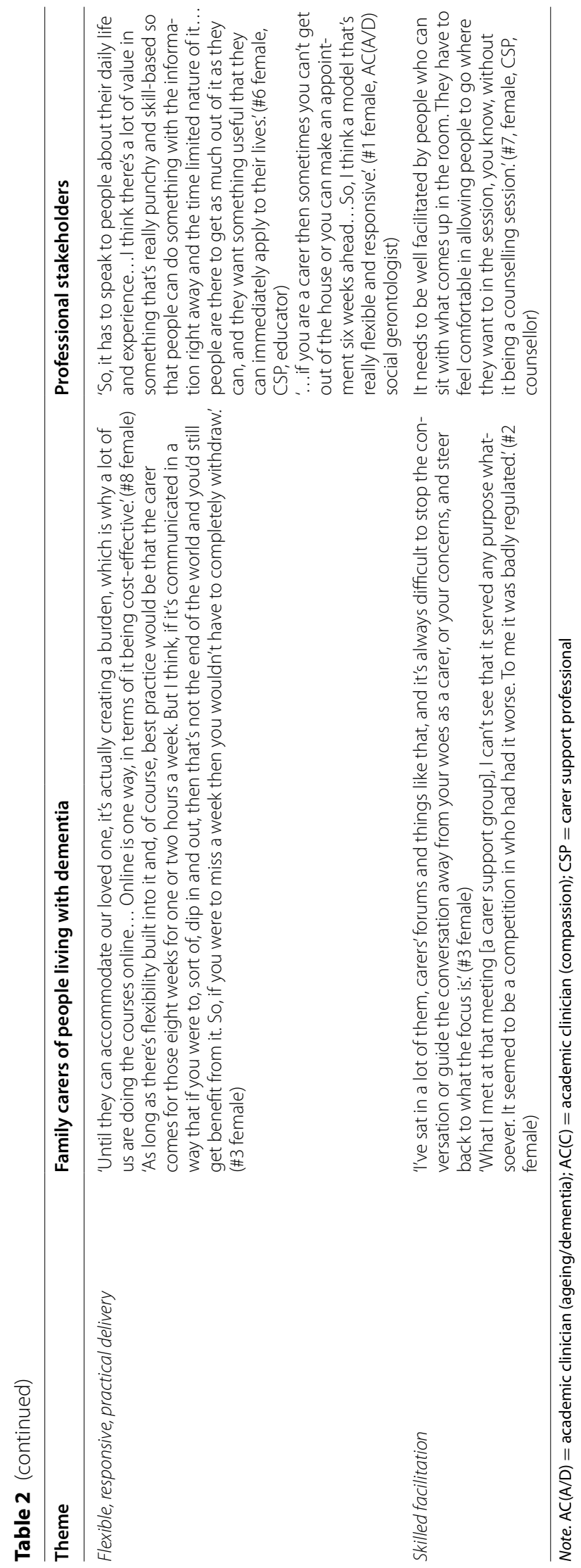


Family carers' individual background characteristics, such as stoicism and gender-, role-, and cultural-based expectations, were considered possible influences on these perceptions of self-compassion.

\section{Realities of Cultivating Self-Compassion}

The constant and demanding nature of being a family carer of a person living with dementia was highlighted by participants as one of the main blocks to carers attending a self-compassion intervention and embedding the practices within everyday life. There was a perception that many family carers were often overwhelmed and, therefore, may either not be open to self-compassion or would view the required activities and practices as another stressor.

Many participants considered family carers to be outward focused in their efforts (i.e., placing the needs of the care recipient first) and, as such, spent little time on inward work on the self. Professional stakeholders also thought that because family carers did not routinely prioritise themselves, self-compassion was not something that family carers would either think of or potentially consider possible for themselves.

\section{Fears of Self-Compassion}

Although the opportunity for self-reflection and the cultivation of self-compassion was seen as a positive thing for emotional health, participants also recognised that this could be challenging for many carers. There was a perception that family carers of people living with dementia often avoided emotional reflection to enable them to continue in the caregiving role and through fear of emotional breakdown. For some family carers of people living with dementia, it was thought that emotional reflection could lead to negative thoughts about their situation and, ultimately, the person they were caring for, which could then lead to feelings of guilt and shame. It was also thought that family carers of people living with dementia could be particularly self-critical and that emotional reflection may exacerbate these feelings (i.e., highlighting that they were not as compassionate to themselves as they should be).

\section{Supporting Attendance and Implementation}

Participants commented that the intervention's success would likely depend on how it was pitched. Although some participants thought it helpful to demonstrate the potential benefits of the intervention for both the carer and the care recipient (i.e., will support you to be a better carer), there was a more common view that to ensure clarity about the intervention's focus, it was important to be explicit that the focus was on the carer personally.
Due to the nature of the caring role, participants stressed that the intervention needed to be as flexible, responsive, and practical as possible. Psychoeducation and practical skills-based learning that was relevant and able to be incorporated into daily life were highlighted. Additionally, with carers limited in their ability to attend sessions due to their role, the mode of delivery was raised. The potential for online delivery (rather than face-to-face) was the main suggestion, although some stakeholders highlighted reduced efficacy and technical issues as considerations, as well as potential issues with recruitment.

Participants acknowledged the importance of having a trained and skilled facilitator to run the intervention group. Many commented on the sometimes-judgmental nature of carers with each other, which, if not facilitated well in group work, could be unproductive. Professional stakeholders also specifically commented on the need for the intervention to be led by a trained mental health professional.

Most participants highlighted the need to consider alternative care provision for the care recipient, particularly if the intervention required in-person attendance. There was a prevailing view against undertaking dyadic group work (i.e., carers and care recipient in the group together), as this may inhibit carers from talking about their feelings and situation. However, one stakeholder had undertaken dyadic work with family carers of people living with dementia within a similar area, and this had been successful.

\section{Stage 2 Intervention Design: Co-Design Group}

Drawing on the themes generated from the in-depth qualitative interviews conducted in Stage 1 and the findings from our earlier evidence- and theory-based activities (see [12, 18, 42, 43]), we developed draft guiding principles and drew on psychological theory to inform the proposed design of the intervention. We then convened a co-design group and presented both aspects to them for discussion, feedback, and/or refinement. Alongside this, the co-design group also assisted in creating caregiver scenarios and personas for use in the intervention. Table 1 provides the background characteristics of the six co-design group members.

\section{Guiding Principles}

Our intervention's guiding features focused primarily on the importance of addressing potential misperceptions, fears, blocks, and resistances to self-compassion and how attendance could be best supported through consideration of issues related to intervention delivery. Co-design group members agreed with the content of the guiding 
principles; however, they felt that the language used to define the key issues, design objectives, and features was unnecessarily complex and not easy for them to understand. Therefore, to ensure that the principles of PPI were upheld throughout the process of the intervention's development (i.e., challenging potential power imbalances between the researchers and co-design members) the language used in the guiding principles was simplified. This was an important step in designing the intervention and ensured that co-design members were able to contribute equally and with confidence to the process. Table 3 provides an overview of the finalised guiding principles, which were iteratively developed and agreed upon in consultation with the co-design group.

\section{Incorporating Psychological Theory}

The knowledge collectively generated during intervention planning inductively identified that it was important for the self-compassion intervention to be presented to family carers of people living with dementia in a way that situated it within the concept of compassion more broadly; addressed misperceptions, fears, blocks, and resistances to self-compassion; and targeted feelings of shame, guilt, and self-criticism. Drawing on the psychological literature, we therefore determined that, rather than developing a novel self-compassion intervention, the needs of family carers of people living with dementia could be best met by tailoring an existing approach: group-based Compassion-Focused Therapy (CFT) [14].

CFT is an integrative approach that aims to develop compassion (both for the self and others) to improve emotional wellbeing by targeting shame and self-criticism [14]. It defines compassion as a motivation that can be directed towards others, from others, or towards the self (self-compassion), and it works explicitly with fears, blocks, and resistances to compassion [14]. Delivered by trained facilitators, the approach has traditionally focused on the dynamic therapeutic process and therefore has not been manualised. However, a standardised, 12-module group CFT manual is under development (Gilbert, Kirby and Petrocchi) and, although not publicly available, a few studies have been given access and successfully tailored the manual for use with specific populations $[49,50]$.

We provided co-design group members with a brief theoretical introduction to CFT and an overview of the 12-module manual, as used and described in the study by Carter et al. [50]. We then asked the group for their thoughts on how CFT aligned with the guiding principles and for their general perceptions of CFT as an intervention for use with family carers of people living with dementia. All co-design members agreed that there was a strong alignment of CFT with the formulated guiding principles and fully supported tailoring the group CFT for our population of interest.

\section{Creation of Caregiver Scenarios and Personas}

In line with the PBA's recommendation to undertake user-centred designed [26], we asked co-design members to create scenarios and personas for use within the intervention that were about any aspect of the guiding principles (i.e., perceptions, fears, blocks, resistances etc.). We presented a working example, and each co-design member worked individually to draft their scenarios. Collectively, fourteen fictional scenarios and personas were created, and these can be used within the developed intervention to help ground the content to the experiences of family carers of people living with dementia. For illustrative purposes, two of the created scenarios are provided below:

The first introduction to self-compassion was during a stressful, demanding and exhausting time in my life and I heard the term and it just sounded so reminiscent of the popular positive pop psychology books, workshops etc. that sound great, take your money and you are no better off.

George was reluctant to join a group to develop his feelings of compassion. He hadn't had a good experience in any of the other carer groups he'd joined, and he felt uneasy about revealing too much of himself to others. He was filled with so much grief at watching the person he loved most in the world fade away. He felt a shadow of the person he was before. He knew the caregiver journey had changed him but how could he explain that within a group where others might judge him?

\section{Discussion}

Within this article, we have described the research activities undertaken in planning and designing a self-compassion intervention for family carers of people living with dementia that used a person-based and co-design approach. By systematically reporting the process we took, we offer insight into planning and designing an intervention that is applicable to both the development of our intervention and the development of complex health interventions generally. Additionally, we also show the value of incorporating in-depth qualitative research and co-design within the intervention development process, with our findings determining that the needs of our population were best met by tailoring an existing intervention rather than developing a novel intervention.

The qualitative research and co-design group we undertook generated knowledge that ensured that the intervention was planned and designed with the needs and preferences of family carers of people living with dementia at its core. Although some of these issues were 


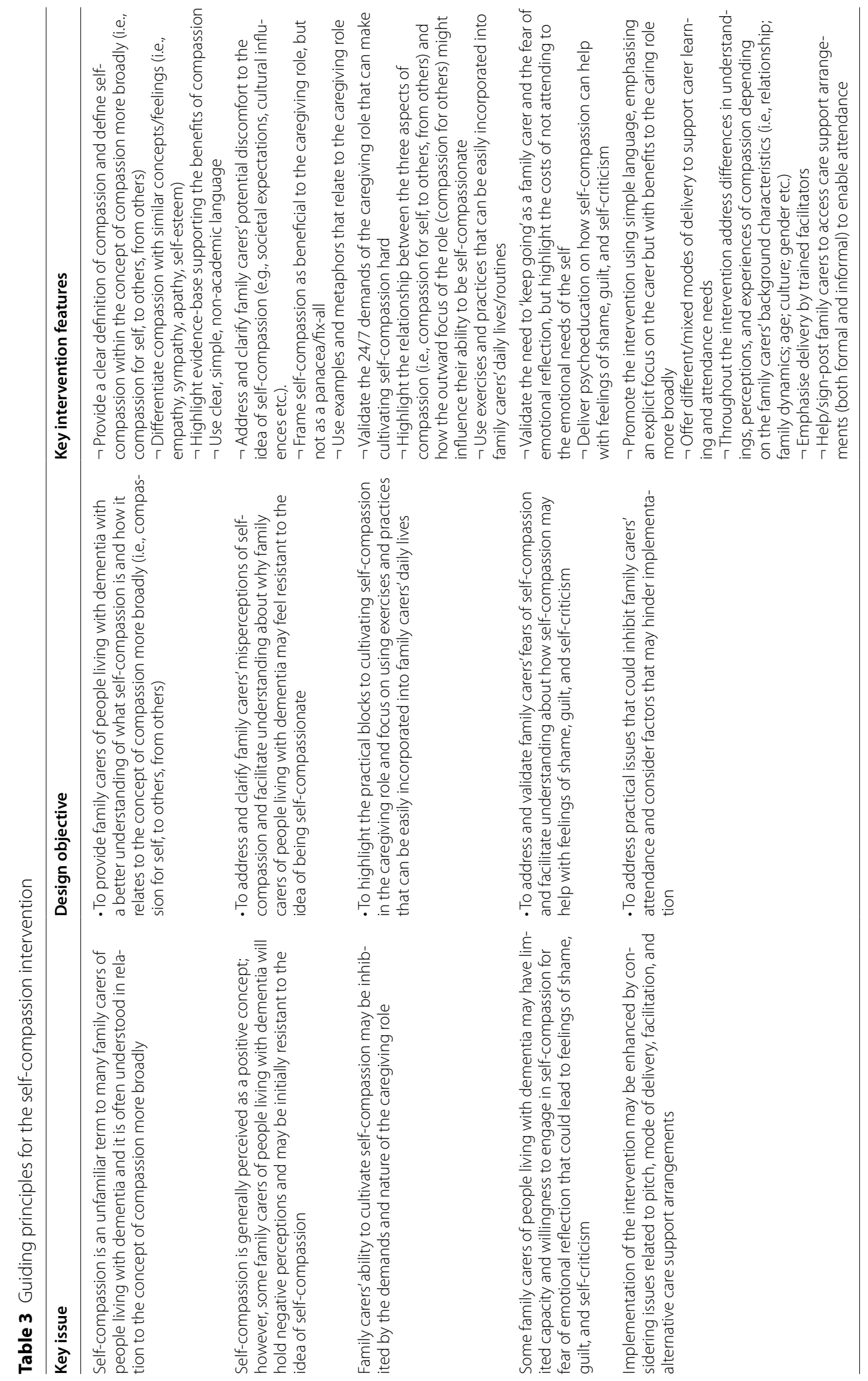


identified in our earlier evidence- and theory-based activities [12, 18, 42-44], without this in-depth qualitative and co-design work, we would not have understood their centrality to the acceptability of the intervention for our population. Specifically, during the initial planning stage, we found that dementia family carers' perceptions of self-compassion aligned with Gilbert's [14] conceptualisation, seeing it situated within the concept of compassion as it relates to self and others. We also found that family carers of people living with dementia had several misperceptions about self-compassion, as well as some fears, blocks, and resistances that could lead to enhanced feelings of shame, guilt, and self-criticism. These findings align with research conducted with various populations (e.g., [51-54]), including family carers of people living with dementia (e.g., $[6,7,55,56])$, and thus supports their importance within our intervention's design. Alongside this, we also found that acceptability of, and likely engagement with, the intervention was influenced by implementation issues, including pitch, method of delivery, facilitation, and alternative care support arrangements. Similar issues were raised as important factors during the recruitment and retention of dementia family carers to a mindfulness-based intervention [57], and therefore indicates their need for consideration within comparable interventions such as ours.

During the design stage, we then used the described findings to determine the intervention's key content and key features to maximise user acceptability and engagement, which involved the formulation of guiding principles, incorporation of psychological theory, and PPI through a process of co-design. In doing so, we identified that the needs of family carers of people living with dementia were keenly aligned with CFT [14] and that, rather than developing a novel intervention, the soon-to-be-published group manual (Gilbert, Kirby and Petrocchi) affords an opportunity to tailor CFT to our population's needs. Specifically, CFT conceptualises self-compassion within compassion more generally; is delivered by a trained facilitator; targets shame and selfcriticism; and works explicitly with fears, blocks, and resistances to self-compassion. To our knowledge, only one small-scale study has been published reporting the outcomes of group-based CFT with family carers of people living with dementia [19]. Importantly, however, this intervention differs from ours, as it was not manualised and was dyadic in its delivery (i.e., involving people living with dementia and their spouses).

\section{Limitations}

First, although our approach to planning and designing the intervention was systematic, it could be regarded as lengthy and limited to situations where resources of both time and funds are adequate. However, the PBA is nonprescriptive in its application [24-26], and researchers can adapt the research activities to meet their projectspecific needs. Second, although we sampled interview participants and co-design members with different characteristics, the majority of our participants were Australian women providing care to either a parent or partner living with dementia. As such, the generalisability of our findings beyond this group is not guaranteed. Third, the lead author conducted all interviews and co-design sessions as part of their doctoral research program. This may have introduced social desirability, with potentially more favourable opinions and preferences expressed to support the lead author. Fourth, due to COVID-19, all PPI was conducted via remote co-design sessions using either videoconferencing or telephone. Although codesign group members informally expressed satisfaction with their involvement in this way, we acknowledge that in-person sessions may have generated a richer experience and data [58].

\section{Future Research}

Building on the knowledge produced during this study, the proposed intervention can progress to the next stages of development and feasibility testing by continuing to use an evidence-based, theory-based, person-based, and co-design approach [24-29, 33, 34]. Next steps will see the guiding principles used to tailor the CFT group manual (once publicly available) for family carers of people living with dementia; all intervention-related documents developed (i.e., information sheets, advertisements etc.); and the intervention's mode of delivery finalised. After this, the acceptability of the intervention's components can be tested using think-aloud techniques with family carers of people living with dementia, professional stakeholders, and co-design group members. To ensure that the intervention is optimised to the needs of our population, this may see iterative changes made to the intervention up until it is deemed ready for acceptability and feasibility evaluation.

\section{Conclusions}

With a recognised evidence-to-practice gap for interventions within gerontology, new and alternative approaches to supporting family carers of people living with dementia should be developed in ways that are systematic and have the needs and preferences of intended users at the centre. This article provides an example of how indepth qualitative research and co-design processes were systematically used to plan and design a self-compassion intervention for family carers of older adults. The approach highlights the potential of using the PBA and co-design in intervention development, with research 
activities determining that the needs of our population were optimally met by tailoring an existing intervention. Further, the systematic reporting of the planning and design process offers useful insight that is applicable to both our intervention and those interested in developing complex health interventions more broadly.

\section{Abbreviations}

CFT: Compassion-Focused Therapy; GUIDED: Guidelines for the Reporting of Intervention Development Checklist; GRIPP2-SF: Guidance for Reporting Involvement of Patients and the Public; MRC: Medical Research Council; PBA: Person-Based Approach; PPI: Patient and Public Involvement.

\section{Supplementary Information}

The online version contains supplementary material available at https://doi. org/10.1186/s12877-022-02754-9.

Additional file 1. GUIDED and GRIPP2-SF Reporting Guideline Checklists. Completed reporting guidelines checklists for GUIDED (guidelines for reporting intervention development studies) and GRIPP2-SF (guidelines for reporting involvement of patients and the public-short form)

\section{Acknowledgements}

We thank the individuals who gave up their time to be involved in this research and the organisations, services, and online support groups who assisted us with recruitment activities.

\section{Authors' contributions}

JM conceived and designed the study, undertook data collection and analysis, and wrote, revised, and finalised the manuscript. WM and AOD supervised the study, advised on aspects of study design, data collection and analysis, and conceptually commented on and revised the manuscript. All authors read and approved the final manuscript.

\section{Funding}

This work was supported by a Griffith University Postgraduate Scholarship awarded to Jenny Murfield. The funder had no role in the design of the study, the collection, analysis and interpretation of data, and preparation and finalisation of the article.

\section{Availability of data and materials}

The datasets generated and/or analysed in this study cannot be made publicly available as participants did not provide consent for their data to be shared. Further details about the data and ethical conditions for access are available from the corresponding author.

\section{Declarations}

\section{Ethics approval and consent to participate}

This study was approved by Griffith University Human Research Ethics Committee (GU ref. no. 2019/481) and performed in accordance with ethical standards as laid down in the 1964 Declaration of Helsinki and its later amendments. Written informed consent to participate was obtained from all participants and co-design group members.

\section{Consent for publication}

Not applicable.

\section{Competing interests}

The authors declare that they have no competing interests.

\section{Author details}

${ }^{1}$ Food \& Mood Centre, IMPACT (Institute for Mental and Physical Health and Clinical Translation), School of Medicine, Deakin University, Geelong,
Australia. ${ }^{2}$ Menzies Health Institute Queensland, Griffith University, Brisbane, Australia. ${ }^{3}$ School of Nursing and Midwifery, Griffith University, Brisbane, Australia. ${ }^{4}$ Griffith Health Group, Griffith University, Brisbane, Australia.

Received: 31 May 2021 Accepted: 24 December 2021

Published online: 14 January 2022

\section{References}

1. Yu DSF, Cheng ST, Wang J. Unravelling positive aspects of caregiving in dementia: An integrative review of research literature. Int J Nurs Stud. 2018;79:1-26 https://doi.org/10.1016/j.ijnurstu.2017.10.008.

2. Merrilees $J$. The impact of dementia on family caregivers: What is research teaching us? Curr Neurol Neurosci Rep. 2016;16(10):88 https://doi.org/10. 1007/s11910-016-0692-z

3. Pinquart $M$, Sorensen S. Differences between caregivers and noncaregivers in psychological health and physical health: A meta-analysis. Psychol Aging. 2003;18(2):250-67 https://doi.org/10.1037/0882-7974.18.2.250

4. Teahan Á, Lafferty A, Cullinan J, Fealy G, O'Shea E. An analysis of carer burden among family carers of people with and without dementia in Ireland. Int Psychogeriatr. 2020:1-12 https://doi.org/10.1017/S104161022 0000769

5. Collins RN, Kishita N. Prevalence of depression and burden among informal care-givers of people with dementia: A meta-analysis. Ageing Soc. 2020;40(11):2355-92 https://doi.org/10.1017/S0144686X19000527.

6. Losada A, Marquez-Gonzalez M, Vara-Garcia C, Gallego-Alberto L, Romero-Moreno R, Pillemer K. Ambivalence and guilt feelings: Two relevant variables for understanding caregivers' depressive symptomatology. Clin Psychol Psychother. 2018;25(1):59-64 https://doi.org/10.1002/ cpp.2116.

7. Martin Y, Gilbert P, McEwan K, Irons C. The relation of entrapment, shame and guilt to depression, in carers of people with dementia. Aging Ment Health. 2006;10(2):101-6 https://doi.org/10.1080/13607860500311953.

8. Teahan Á, Lafferty A, McAuliffe E, Phelan A, O'Sullivan L, O'Shea D, et al. Psychosocial interventions for family carers of people with dementia: A systematic review and meta-analysis. J Aging Health. 2020;32(9):1198213 https://doi.org/10.1177/0898264319899793.

9. Kishita N, Hammond L, Dietrich CM, Mioshi E. Which interventions work for dementia family carers? An updated systematic review of randomized controlled trials of carer interventions. Int Psychogeriatr. 2018:30(11):1679-96 https://doi.org/10.1017/S1041610218000947.

10. Wiegelmann H, Speller S, Verhaert L-M, Schirra-Weirich L, Wolf-Ostermann K. Psychosocial interventions to support the mental health of informal caregivers of persons living with dementia - a systematic literature review. BMC Geriatr. 2021;21(1):94 https://doi.org/10.1186/ s12877-021-02020-4.

11. Cheng ST, Au A, Losada A, Thompson LW, Gallagher-Thompson D. Psychological interventions for dementia caregivers: What we have achieved, what we have learned. Current Psychiatry Reports. 2019;21(7):59 https:// doi.org/10.1007/s11920-019-1045-9.

12. Murfield J, Moyle W, O'Donovan A. Mindfulness- and compassion-based interventions for family carers of older adults: A scoping review. Int J Nurs Stud. 2021:103495 https://doi.org/10.1016/j.jinurstu.2019.103495.

13. Neff K. Self-compassion: An alternative conceptualization of a healthy attitude toward oneself. Self Identity. 2003;2(2):85-101 https://doi.org/10. 1080/15298860390129863.

14. Gilbert P. The origins and nature of compassion focused therapy. Br J Clin Psychol. 2014;53(1):6-41 https://doi.org/10.1111/bjc.12043.

15. Zessin U, Dickhäuser O, Garbade S. The relationship between self-compassion and well-being: A meta-analysis. Applied Psychology: Health and Well-Being. 2015;7(3):340-64 https://doi.org/10.1111/aphw.12051.

16. Kirby JN, Tellegen CL, Steind I SR. A meta-analysis of compassion-based interventions: Current state of knowledge and future directions. Behav Ther. 2017;48(6):778-92 https://doi.org/10.1016/j.beth.2017.06.003.

17. Lloyd J, Muers J, Patterson T, Marczak M. Self-compassion, coping strategies, and caregiver burden in caregivers of people with dementia. Clin Gerontol. 2019;42(1):47-59 https://doi.org/10.1080/07317115.2018.14611 62.

18. Murfield J, Moyle W, O'Donovan A, Ware R. The role of self-compassion, dispositional mindfulness, and emotion regulation in the psychological 
health of family carers of older adults Clin Gerontol. 2020:1-13 https:// doi.org/10.1080/07317115.2020.1846650.

19. Collins RN, Gilligan LJ, Poz R. The evaluation of a compassion-focused therapy group for couples experiencing a dementia diagnosis. Clin Gerontol. 2018:41(5):474-86 https://doi.org/10.1080/07317115.2017.13978 30.

20. Gaugler JE, Jutkowitz E, Shippee TP, Brasure M. Consistency of dementia caregiver intervention classification: An evidence-based synthesis. Int Psychogeriatr. 2017;29(1):19-30 https://doi.org/10.1017/S104161021 6001514

21. Donkers HW, Van der Veen DJ, Teerenstra S, Vernooij-Dassen MJ, Nijhuisvander Sanden MWG, Graff MJL. Evaluating the social fitness programme for older people with cognitive problems and their caregivers: Lessons learned from a failed trial. BMC Geriatr. 2018;18(1):237 https://doi.org/10 1186/s12877-018-0927-8.

22. Slattery P, Saeri AK, Bragge P. Research co-design in health: A rapid overview of reviews. Health Res Policy Syst. 2020;18(1):17 https://doi.org/10, 1186/s12961-020-0528-9.

23. Michie S, Fixsen D, Grimshaw JM, Eccles MP. Specifying and reporting complex behaviour change interventions: The need for a scientific method. Implementation Science. 2009;4(1):40 https://doi.org/10.1186/ 1748-5908-4-40.

24. Morrison L, Muller I, Yardley L, Bradbury K. The person-based approach to planning, optimising, evaluating and implementing behavioural health interventions. The European Health Psychologist. 2018;20(3):464-9

25. Yardley L, Ainsworth B, Arden-Close E, Muller I. The person-based approach to enhancing the acceptability and feasibility of interventions. Pilot and Feasibility Studies. 2015;1 (1):37 https://doi.org/10.1186/ s40814-015-0033-z.

26. Yardley L, Morrison L, Bradbury K, Muller I. The person-based approach to intervention development: Application to digital health-related behavior change interventions. J Med Internet Res. 2015;17(1):e30 https://doi.org/ 10.2196/jmir.4055.

27. Langley J, Wolstenholme D, Cooke J.'Collective making' as knowledge mobilisation: The contribution of participatory design in the co-creation of knowledge in healthcare. BMC Health Serv Res. 2018;18(1):585 https:// doi.org/10.1186/s12913-018-3397-y.

28. National Health and Medical Research Council. Statement on consumer and community involvement in health and medical research. Canberra, Australia: National Health and Medical Research Council; 2016.

29. INVOLVE. Briefing notes for researchers: Involving the public in NHS, public health, and social care research. Eastleigh, UK: INVOLVE; 2012

30. Dale J, Loew J, Nanton V, Grason Smith G. Coproduction of a theorybased digital resource for unpaid carers (The Care Companion): Mixedmethods study. JMIR Aging. 2018;1(1):e1 https://doi.org/10.2196/aging. 9025.

31. Lawrence V, Kimona K, Howard RJ, Serfaty MA, Wetherell JL, Livingston $\mathrm{G}$, et al. Optimising the acceptability and feasibility of acceptance and commitment therapy for treatment-resistant generalised anxiety disorder in older adults. Age Ageing. 2019;48(5):741-50 https://doi.org/10.1093/ ageing/afz082.

32. Essery R, Pollet S, Smith K, A., Mowbray F, Slodkowska-Barabasz J, Denison-Day J, et al. Planning and optimising a digital intervention to reduce older adults' cognitive decline. Pilot and Feasibility Studies. 2021;7(1):158 https://doi.org/10.1186/s40814-021-00884-2.

33. Craig P, Dieppe P, Macintyre S, Michie S, Nazareth I, Petticrew M. Developing and evaluating complex interventions: The new Medical Research Council guidance. BMJ. 2008;337:a1655 https://doi.org/10.1136/bmj. a1655.

34. Craig P, Dieppe P, Macintyre S, Michie S, Nazareth I, Petticrew M. Developing and evaluating complex interventions: The new Medical Research Council guidance. Int J Nurs Stud. 2013;50(5):587-92 https://doi.org/10. 1016/j.jinurstu.2012.09.010.

35. Muller I, Santer M, Morrison L, Morton K, Roberts A, Rice C, et al. Combining qualitative research with PPI: Reflections on using the personbased approach for developing behavioural interventions. Research Involvement and Engagement. 2019;5(1):34 https://doi.org/10.1186/ s40900-019-0169-8

36. Greenhalgh T, Hinton L, Finlay T, Macfarlane A, Fahy N, Clyde B, et al. Frameworks for supporting patient and public involvement in research:
Systematic review and co-design pilot. Health Expect. 2019;22(4):785801 https://doi.org/10.1111/hex.12888.

37. Bethell J, Commisso E, Rostad HM, Puts M, Babineau J, Grinbergs-Saull $A$, et al. Patient engagement in research related to dementia: A scoping review. Dementia. 2018;17(8):944-75 https://doi.org/10.1177/14713 01218789292.

38. Wang G, Marradi C, Albayrak A, van der Cammen TJM. Co-designing with people with dementia: A scoping review of involving people with dementia in design research. Maturitas. 2019;127:55-63 https://doi.org/ 10.1016/j.maturitas.2019.06.003

39. Goeman DP, Corlis M, Swaffer K, Jenner V, Thompson JF, Renehan E, et al. Partnering with people with dementia and their care partners, aged care service experts, policymakers and academics: A co-design process. Australas J Ageing. 2019;38(S2):53-8 https://doi.org/10.1111/ajag.12635.

40. Duncan E, O'Cathain A, Rousseau N, Croot L, Sworn K, Turner KM, et al. Guidance for reporting intervention development studies in health research (GUIDED): An evidence-based consensus study. BMJ Open. 2020;10(4):e033516 https://doi.org/10.1136/bmjopen-2019-033516.

41. Staniszewska S, Brett J, Simera I, Seers K, Mockford C, Goodlad S, et al. GRIPP2 reporting checklists: Tools to improve reporting of patient and public involvement in research. BMJ. 2017;358:j3453 https://doi.org/10. 1136/bmj.j3453.

42. Murfield J, Moyle W, Jones C, O'Donovan A. Self-compassion, health outcomes, and family carers of older adults: An integrative review. Clin Gerontol. 2020;43(5):485-98 https://doi.org/10.1080/07317115.2018. 1560383.

43. Murfield J, Moyle W, O'Donovan A. Self-compassion as an applicable intervention target for family carers of older adults: A conceptual commentary. Int J Geriatr Psychiatry. 2020;35(4):376-83 https://doi.org/10. 1002/gps.5257.

44. Murfield J, Moyle W, O'Donovan A. Experiences of compassion among family carers of older adults: Qualitative content analysis of survey freetext comments. Scand J Caring Sci. 2021:1-10 https://doi.org/10.1111/ scs.13010.

45. Braun V, Clarke V. Reflecting on reflexive thematic analysis. Qual Res Sport Exerc Health. 2019;11(4):589-97 https://doi.org/10.1080/2159676X.2019. 1628806.

46. Braun V, Clarke V. Using thematic analysis in psychology. Qual Res Psychol. 2006;3(2):77-101 https://doi.org/10.1191/1478088706qp063oa.

47. Braun V, Clarke V. One size fits all? What counts as quality practice in (reflexive) thematic analysis? Qual Res Psychol. 2021;18(3):328-52 https:// doi.org/10.1080/14780887.2020.1769238.

48. Braun V, Clarke V. To saturate or not to saturate? Questioning data saturation as a useful concept for thematic analysis and sample-size rationales. Qual Res Sport Exerc Health. 2021;13(2):201-16 https://doi.org/10.1080/ 2159676X.2019.1704846.

49. Fox J, Cattani K, Burlingame GM. Compassion focused therapy in a university counseling and psychological services center: A feasibility trial of a new standardized group manual. Psychotherapy Res J Soc Psychother Res. 2021;31(4):419-31. https://doi.org/10.1080/10503307.2020.1783708.

50. Carter A, Gilbert P, Kirby JN. Compassion-focused therapy for body weight shame: A mixed methods pilot trial. Clin Psychol Psychother. 2021;28(1):93-108 https://doi.org/10.1002/cpp.2488.

51. Gilbert P. Compassion focused therapy: Distinctive features. UK: Taylor and Francis Ltd; 2010

52. Gilbert P, McEwan K, Matos M, Rivis A. Fears of compassion: Development of three self-report measures. Psychol Psychother. 2011;84(3):239-55 https://doi.org/10.1348/147608310X526511.

53. McLean L, Bambling M, Steindl SR. Perspectives on self-compassion from adult female survivors of sexual abuse and the counselors who work with them. Journal of Interpersonal Violence. 2021;36(9-10):NP4564-NP87 https://doi.org/10.1177/0886260518793975.

54. Pauley G, McPherson S. The experience and meaning of compassion and self-compassion for individuals with depression or anxiety. Psychol Psychother. 2010;83(Pt 2):129-43 https://doi.org/10.1348/147608309X 471000.

55. Gonyea JG, Paris R, de Saxe Zerden L. Adult daughters and aging mothers: The role of guilt in the experience of caregiver burden. Aging Ment Health. 2008;12(5):559-67 https://doi.org/10.1080/13607860802343027. 
56. Lacey J, Hiskey S, Andrews L. Compassion focused expressive writing (CFExW) among carers of people with dementia: Some reflections. FPOP Bulletin. 2017; July No.139:32-7

57. Whitebird RR, Kreitzer MJ, Lewis BA, Hanson LR, Crain AL, Enstad CJ, et al. Recruiting and retaining family caregivers to a randomized controlled trial on mindfulness-based stress reduction. Contemp Clin Trials. 2011;32(5):654-61 https://doi.org/10.1016/j.cct.2011.05.002.

58. Krouwel M, Jolly K, Greenfield S. Comparing Skype (video calling) and in-person qualitative interview modes in a study of people with irritable bowel syndrome - an exploratory comparative analysis. BMC Med Res Methodol. 2019;19(1):219 https://doi.org/10.1186/s12874-019-0867-9.

\section{Publisher's Note}

Springer Nature remains neutral with regard to jurisdictional claims in published maps and institutional affiliations.

- fast, convenient online submission

- thorough peer review by experienced researchers in your field

- rapid publication on acceptance

- support for research data, including large and complex data types

- gold Open Access which fosters wider collaboration and increased citations

- maximum visibility for your research: over $100 \mathrm{M}$ website views per year

At BMC, research is always in progress.

Learn more biomedcentral.com/submissions 